\title{
GERMINAÇÃO DE SEMENTES DE ESPÉCIES MEDICINAIS DO RIO GRANDE DO SUL: BROMELIA ANTIACANTAHA BERT., CUPHEA CARTHAGENENSIS (JACQ.) MACBRIDE E TALINUM PATENS (JACQ.) WILLDENOW
}

\author{
Shirley G. T. da Rosa ${ }^{1}$ \\ Alfredo Gui Ferreira ${ }^{1}$
}

Recebido em 28/01/1999. Aceito em 27/07/1999

\begin{abstract}
RESUMO - (Germinação de sementes de espécies medicinais do Rio Grande do Sul: Bromelia antiacantha Bert., Cuphea carthagenensis (Jacq.) Macbride e Talinum patens (Jacq.)Willdenow). As espécies estudadas são ervas de expressiva dispersão no Estado do Rio Grande do Sul e com uso difundido na farmacopéia popular. Estudou-se a influência de diferentes temperaturas constantes $\left(10,15,20,25,30\right.$ e $35^{\circ} \mathrm{C}$ ) sob condição de escuro ou de luz (fotoperíodo: luz/escuro $8 \mathrm{~h} / 16 \mathrm{~h}$ ) e as temperaturas alternadas $\left(10-20,15-25,20-30\right.$ e $\left.25-35^{\circ} \mathrm{C}\right)$ sobre o comportamento germinativo das sementes. As sementes não germinadas foram submetidas ao teste de tetrazólio. Sementes de $B$. antiacantha germinaram acima de $80 \%$ nas temperaturas constantes de 25,30 e $35^{\circ} \mathrm{C}$ na luz e no escuro. Abaixo de $25^{\circ} \mathrm{C}$, as sementes apresentaram dormência relativa. No armazenamento por um ano sob condições ambientais $\left(25^{\circ} \mathrm{C}\right)$ e sob refrigeração $\left(5^{\circ} \mathrm{C}\right)$, a viabilidade das sementes foi mantida em $90 \%$. Sementes de $C$. carthagenensis germinaram acima de $80 \%$ nas temperaturas alternadas. Em temperaturas constantes, a germinação foi nula, mas as sementes estavam viáveis. Em $T$. patens constatou-se acima de $50 \%$ se sementes deterioradas e a germinabilidade foi semelhante nas temperaturas constantes em relação às alternadas. As três espécies podem ser propagadas através de sementes, se as condiçōes de temperatura apropriadas forem observadas.
\end{abstract}

Palavras-chave - Bromelia, Cuphea, Talinum, temperatura, luz, germinação

\begin{abstract}
Seed germination of medicinal plants of Rio Grande do Sul State, Brazil: Bromelia antiacantha Bert., Cuphea carthagenensis (Jacq.) Macbride and Talinum patens (Jacq.) Willdenow). These species of herbs are well distributed over all Rio Grande do Sul State, and are used to prepare medicine for the human population. The influence of constant temperatures $\left(10^{\circ} \mathrm{C} ; 15^{\circ} \mathrm{C} ; 20^{\circ} \mathrm{C}\right.$; $25^{\circ} \mathrm{C} ; 30^{\circ} \mathrm{C}$ and $35^{\circ} \mathrm{C}$ ) on the germination of seeds during a photoperiod of 8 hours, was analyzed. Experiments were also performed in darkness, and at alternating temperatures $\left(10-20^{\circ} \mathrm{C} ; 15-25^{\circ} \mathrm{C}\right.$; $20-30^{\circ} \mathrm{C} ; 25-35^{\circ} \mathrm{C}$ ). Seeds that did not germinate were submitted to the tetrazolium test. Over $80 \%$ of the seeds of $B$. antiacantha germinated at constant temperatures of $25^{\circ}, 30^{\circ}$ or $35^{\circ} \mathrm{C}$, both in light and darkness. Below $25^{\circ} \mathrm{C}$, the seeds presented relative dormancy. Storage during one year under laboratory conditions $\left(25^{\circ} \mathrm{C}\right)$, or at a low temperature $\left(5^{\circ} \mathrm{C}\right)$ allowed for $90 \%$ of seed viability. Over $80 \%$ of the seeds of $C$. carthagensis germinated at alternating temperatures, but no germination was
\end{abstract}

1 Departamento de Botânica, Laboratório de Fisiologia Vegetal, IB,Universidade Federal do Rio Grande do Sul, CEP 91509-900, Porto Alegre, RS, Brasil 
observed at constant temperatures, although the seeds remained viable. T. patens presented over $50 \%$ of deteriorated seeds and final germination was similar at constant and alternating temperatures. The three species can be propagated through seeds, if the appropriate temperature requirements are observed for each of them.

Key words - Bromelia, Cuphea, Talinum, light, temperature, germination

\section{Introdução}

Bromelia antiacantha Bert. (Bromeliaceae), Cuphea carthagenensis (Jacq.) Macbride (Lythraceae) e Talinum patens (Jacq.) Willdenow (Portulacaceae) são espécies de expressiva dispersão no Estado do Rio Grande do Sul. Seus habitats variam desde floresta de galerias (B. antiacantha) a campos abertos ( $C$. carthagenensis) ou em orla de matas (T. patens), mas todas ocorrem preferencialmente em solos úmidos.

As três espécies possuem escassas informaçōes farmacológicas sobre seus compostos ativos mas têm uso difundido popularmente: $B$. antiacantha ("banana-domato") possui frutos do tipo baga, amarelos, com atividade expectorante e anti-helmíntica (Reitz 1983); C. carthagenensis ("sete-sangrias") cujos ramos têm atividade hipotensora e depressiva do sistema nervoso, tem uso popular como calmante (Lopes 1997); $T$. patens ("João-Gomes") é citada por Magalhães (1997) por suas folhas serem vermífugas.

A pesquisa de plantas medicinais é interdisciplinar, envolvendo farmacognosia, etnobiologia, agronomia, ecologia e botânica. A segura caracterização da espécie tida como medicinal, bem como a determinação de sua forma de propagação, é uma das etapas iniciais e decisivas para o estabelecimento de seu cultivo. A propagação pode ocorrer de dois modos: vegetativamente e/ou por sementes.

Segundo Bewley \& Black (1982), a germinação de uma semente começa com a absorção de água (embebição) e termina com o inicío do alongamento do eixo embrionário, que geralmente é a radícula, e sua conseqüente extrusão dos envoltórios. Em geral, a germinabilidade de uma semente depende, entre os fatores principais, de água, oxigênio, temperatura e luz.

A luz e a temperatura são os fatores de maior importância na promoção da germinação de sementes dormentes em solos úmidos. Para muitas espécies, se fornecidas as condições ideais de luz e umidade, a temperatura predominante do solo determina quantas sementes germinarão em uma amostra, bem como a velocidade de germinação (Heydecker 1977).

Conforme Labouriau (1983), os limites extremos de temperatura e germinação fornecem informações de interesse biológico e ecológico, sendo que sementes de diferentes espécies apresentam faixas distintas de temperatura para a germinação. Dentro destas faixas, pode ser considerada como ótima aquela na qual a mais alta percentagem de germinação é obtida, dentro do menor espaço de tempo. A mínima e a máxima seriam consideradas, respectivamente, como a mais baixa e a mais alta temperatura onde a germinação ocorre. Sementes de muitas espécies germinam melhor sob temperaturas alternadas do que sob temperaturas constantes (Koller 1972). 
Sementes podem ser divididas em grupos que germinam somente quando expostas à luz, as que germinam somente se não receberam luz e aquelas que são indiferentes à ausência ou à presença de luz (Mayer \& Poljakoff-Mayber 1989).

Muitas espécies cultivadas são indiferentes à luz para germinar, entretanto, o estímulo luminoso é bastante variável em sementes de várias espécies selvagens (Mayer \& Poljakoff-Mayber 1989). Labouriau (1983) cita que para muitas espécies a presença de luz aumenta a germinabilidade (fotoblastismo positivo), enquanto em outras espécies, a germinabilidade é menor na presença de luz do que no escuro (fotoblastismo negativo). Em apenas 5\% das espécies estudadas a germinação foi indiferente a luz.

B. antiacantha, $C$. carthagenensis e $T$. patens possuem amplo uso medicinal e são obtidas por extrativismo, o que pode prejudicar a regeneração natural sem plano de manejo adequado. Esta situação é agravada no caso de $B$. antiacantha, por ser espécie monocárpica perene (Eira 1997). Tendo em vista a ausência e a importância de estudos sobre a propagação de espécies nativas de expressão medicinal, o presente trabalho objetivou avaliar a influência da luz e da temperatura sobre o comportamento germinativo das espécies acima mencionadas.

\section{Material e métodos}

Foram coletados frutos de uma população de $B$. antiacantha em mata de galeria, em abril/1997 e frutos de $C$. carthagenensis e $T$. patens em fevereiro/1998 em mancha de campo úmido e em orla de mata, respectivamente.

Os frutos foram beneficiados, sendo analisados o teor de umidade $\left(103^{\circ} \mathrm{C} \pm 2\right.$ por 24 horas) e o peso de mil sementes (PMS), segundo as Regras de Análises para Sementes (Brasil 1993). As sementes foram desinfestadas com hipoclorito de sódio a $1 \%$ de cloro ativo, por 10min e a seguir enxaguadas em água esterilizada. Sob condições assépticas, as sementes foram espalhadas aleatoriamente em placas de petri de $9 \mathrm{~cm}$ diâm., contendo aproximadamente $20 \mathrm{ml}$ de ágar a $1 \%$.

Os testes de germinação foram realizados em câmaras B.O.D., marca FANEM, com temperaturas constantes $\left( \pm 2^{\circ} \mathrm{C}\right.$ ) de $10^{\circ}, 15^{\circ} \mathrm{C}$ (ausência de luz), $20^{\circ}, 25^{\circ}, 30^{\circ} \mathrm{e}$ $35^{\circ} \mathrm{C}$ (ausência e presença de luz). Também foram avaliadas as temperaturas alternadas de $10-20^{\circ} \mathrm{C}, 15-25^{\circ} \mathrm{C}, 20-30^{\circ} \mathrm{C}$ e $25-35^{\circ} \mathrm{C}$, sendo a temperatura mais alta na luz. As câmaras eram providas de lâmpadas fluorescentes e com fotoperíodo de $9 \mathrm{~h}$ de luz. $\mathrm{O}$ fluxo de radiação aproximado foi de $50 \mu \mathrm{mol} \mathrm{m}^{-2} \mathrm{~s}^{-1}$, e a ausência de luz foi obtida pelo envolvimento das placas de petri em duas folhas de papel aluminizado.

$\mathrm{O}$ delineamento experimental foi completamente casualizado com quatro repetições de 25 sementes por tratamento. Em B. antiacantha, o teste foi conduzido durante 42 dias, enquanto para $C$. carthagenensis e $T$. patens, durante 30 dias, ambos com observações a cada três dias. A avaliação no escuro foi feita com auxílio de lâmpada verde de segurança (Labouriau \& Costa 1976). O tempo médio de germinação foi calculado de acordo com Ferreira (1977). O critério de germinação foi a emissão da radícula (no mínimo a metade do comprimento da semente). Os dados foram submetidos a ANOVA e as médias comparadas pelo teste de Duncan (Sokal \& Rohlf 1981), usando o programa SANEST (Zonta \& Machado sd). 
Nas sementes não germinadas utilizou-se o teste de viabilidade de tetrazólio (sal trifenil-cloreto de tetrazólio a $1 \%$ em solução aquosa), o qual avalia a atividade de enzimas do grupo desidrogenase (Grabe 1970).

\section{Resultados}

Sementes de B. antiacantha possuem teor de umidade de $26 \%$ e PMS de $40 \mathrm{~g}, C$. carthagenensis de $9 \%$ e PMS de $0,6 \mathrm{~g}$, enquanto em $T$. patens, o teor de umidade é de $7,5 \%$ e o PMS de $0,24 \mathrm{~g}$. As sementes da primeira espécie germinaram acima de $70 \%$ nas temperaturas alternadas de $25-35^{\circ} \mathrm{C} \mathrm{e} 20-30^{\circ} \mathrm{C}$ (Tab. 1). Em temperaturas constantes de $25^{\circ}, 30^{\circ}$ e $35^{\circ} \mathrm{C}$, tanto na ausência como na presença de luz, as sementes germinaram acima de $80 \%$ (Fig. $1 \mathrm{~A} \mathrm{e} 2 \mathrm{~A}$ ). O processo germinativo em $B$. antiacantha é retardado na luz contínua, quando se compara com os resultados dos tratamentos no escuro (Fig. 1-2) ou em temperaturas alternadas (Tab. 1). Abaixo de $20^{\circ} \mathrm{C}$ ou quando uma das temperaturas do par foi abaixo desta $\left(15-25^{\circ} \mathrm{C}\right.$, por exemplo) não ocorreu germinação (Tab. 1). As sementes não germinadas e submetidas ao teste de tetrazólio revelaram viabilidade acima de $75 \%$, percentual semelhante ao de germinação em areia obtido em temperaturas alternadas de $20-30^{\circ} \mathrm{C}$ (resultados não apresentados). No armazenamento, sementes de B. antiacantha mantiveram-se viáveis, apresentando $90 \%$ de germinação após período de um ano em ambiente de laboratório $\left(25^{\circ} \mathrm{C}\right)$ e em refrigerador $\left(5^{\circ} \mathrm{C}\right)$.

Tabela 1. Sementes de B. antiacantha, C. carthagenensis e T. patens submetidas a temperaturas alternadas. germinadas (A); viáveis pelo teste do tetrazólio (B).

\begin{tabular}{lrrrrrr}
\hline Espécie & \multicolumn{2}{c}{ B. antiacantha } & \multicolumn{2}{c}{ C. carthagenensis } & \multicolumn{2}{c}{ T. patens } \\
Temperaturas alternadas & A & B & A & B & A & B \\
\hline & $(\%)$ & $(\%)$ & $(\%)$ & $(\%)$ & $(\%)$ & $(\%)$ \\
$10-20^{\circ} \mathrm{C}$ & $0 \mathrm{c}$ & 82 & $99 \mathrm{a}$ & 99 & $24 \mathrm{a}$ & 35 \\
$15-25^{\circ} \mathrm{C}$ & $0 \mathrm{c}$ & 82 & $95 \mathrm{ab}$ & 95 & $30 \mathrm{a}$ & 41 \\
$20-30^{\circ} \mathrm{C}$ & $70 \mathrm{~b}$ & 86 & $87 \mathrm{ab}$ & 87 & $42 \mathrm{a}$ & 52 \\
$25-35^{\circ} \mathrm{C}$ & $88 \mathrm{a}$ & 93 & $80 \mathrm{~b}$ & 90 & $42 \mathrm{a}$ & 54 \\
Média geral & & 86 & & 93 & & 45,5 \\
\hline
\end{tabular}

Nas colunas, médias seguidas por letras distintas diferem entre si ao nível de $5 \%$ pelo teste de Duncan.

As sementes de $C$. carthagenensis necessitam alternância de temperatura para germinar. Nas temperaturas de $10-20^{\circ} \mathrm{C}, 15-25^{\circ} \mathrm{C}, 20-30^{\circ} \mathrm{C}$ e $25-35^{\circ} \mathrm{C}$ houve 99,95 , 87 e $80 \%$ de germinação, respectivamente (Tab. 1). A quase totalidade das sementes não germinou em temperaturas constantes, porém mostraram-se viáveis quando submetidas ao teste de viabilidade de tetrazólio (dados não apresentados).

Em T. patens ocorre produção de grande número de sementes. Estas apresentaram baixo percentual de germinação nas temperaturas constantes, tanto em luz constante quanto no escuro (Fig. 3A e 4A). Embora a percentagem de germinação a $30^{\circ} \mathrm{C}$ (Fig. 3A) tenha sido mais elevada que no escuro (Fig. 4A), o menor tempo médio de germinação foi obtido pelo tratamento no escuro nesta temperatura do que na luz (Fig. $3 \mathrm{~B}$ e $4 \mathrm{~B})$. Nas temperaturas alternadas de $10-20^{\circ} \mathrm{C}$ e $15-25^{\circ} \mathrm{C}$ houve retardo na germinação, e germinabilidade mais baixa, ainda que não estatisticamente significativas, do que nos termoperíodos de $20-30^{\circ} \mathrm{C}$ e $25-35^{\circ} \mathrm{C}$ (Tab. 1). As sementes apresentaram baixa viabilidade, constatando-se mais de $50 \%$ de sementes deterioradas. 


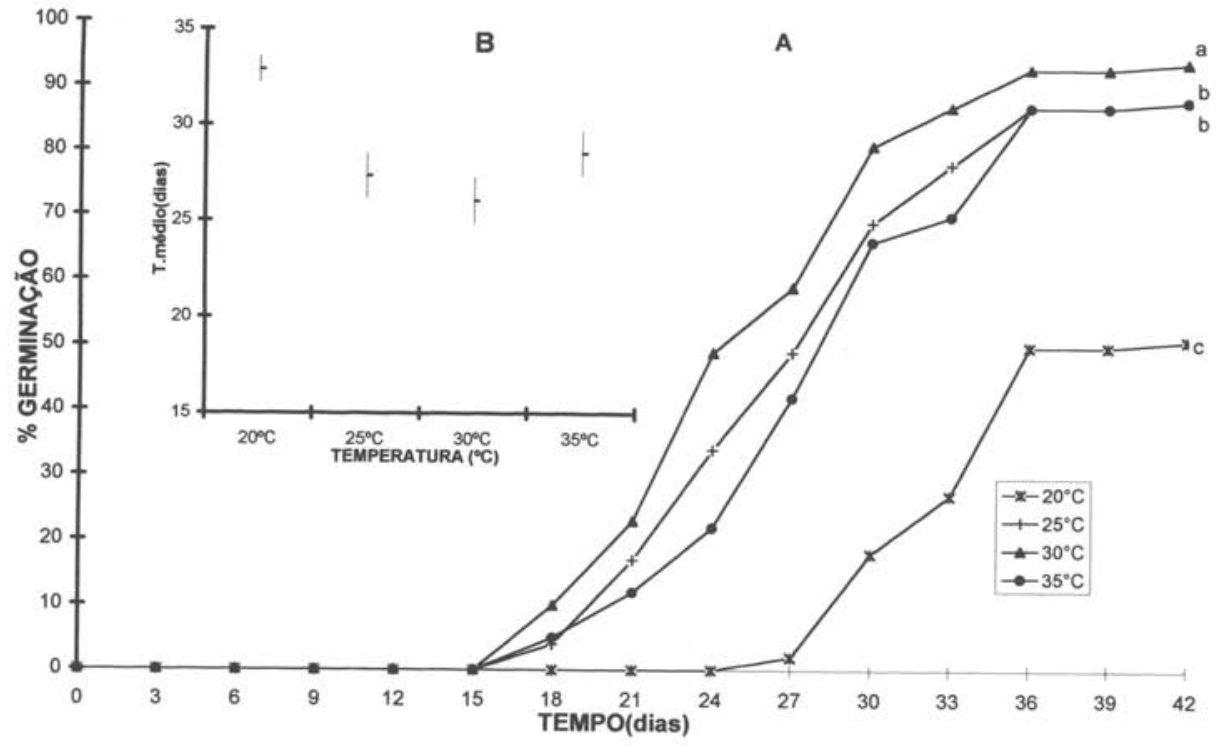

Figural A. Curvas de percentagem acumulada de germinação de sementes de Bromelia antiacantha em temperaturas constantes na presença de luz. Médias seguidas por letras distintas diferem entre si ao nível de $5 \%$. B. Tempo médio de germinação.

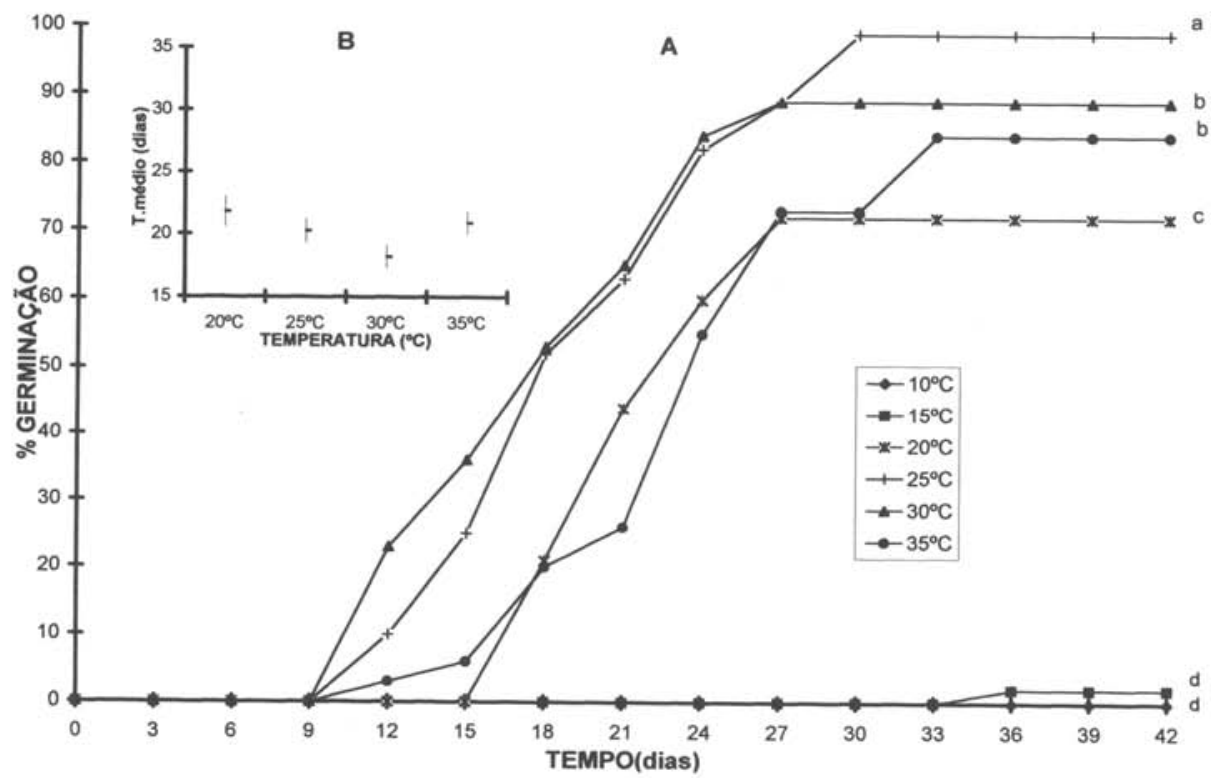

Figura $2 \mathrm{~A}$. Curvas de percentagem acumulada da germinação de sementes de Bromelia antiacantha em temperaturas constantes no escuro. Médias seguidas por letras distintas diferem entre si ao nível $5 \%$. B. Tempo médio de germinação. 


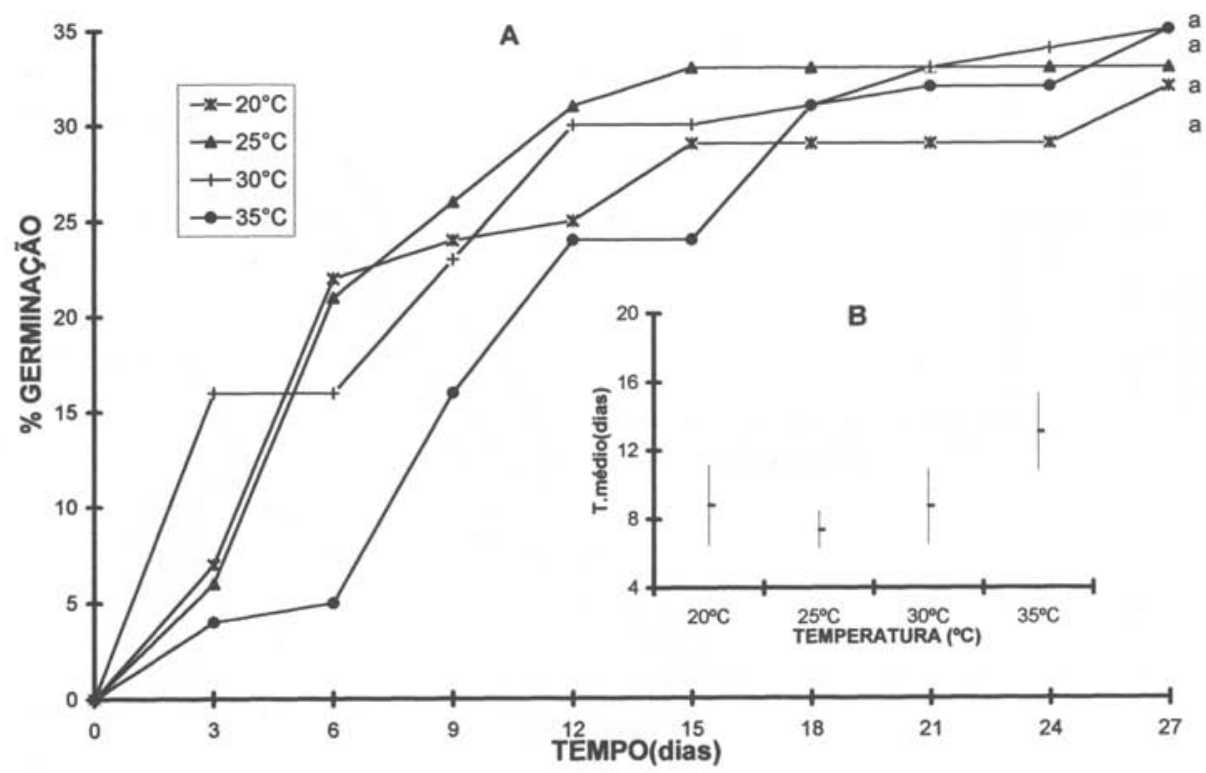

Figura 3. A. Curvas de percentagem acumulada da germinação de sementes de Talinum patens em temperaturas constantes na presença de luz. Médias seguidas por letras distintas diferem entre si ao nível de $5 \%$. B. Tempo médio de germinação.

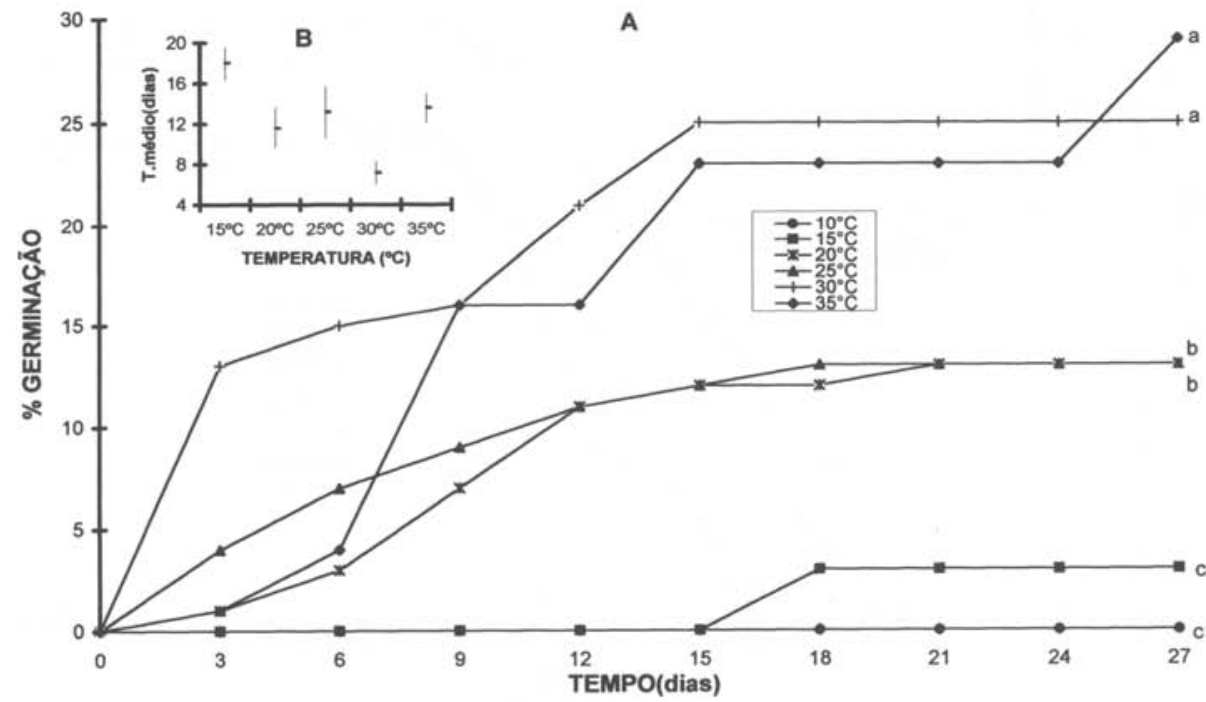

Figura 4. A. Curvas de percentagem acumulada da germinação de sementes de Talinum patens em temperaturas constantes no escuro. Médias seguidas por letras distintas diferem entre si ao nível de $5 \%$. B. Tempo médio de germinação. 


\section{Discussão}

A promoção da germinação de sementes por alternância de temperaturas é fenômeno relativamente comum (Koller 1972; Thompson 1974a; Ghersa et al. 1992; Benech-Arnold \& Sanchez 1995). Para as sementes de C. carthagenensis, a alternância de temperatura é indispensável para ocorrer a germinação. $\mathrm{O}$ aumento das flutuações diárias de temperatura indicam para as sementes que estas devem estar próximas à superfície do solo e, portanto, mais aptas a ter sucesso na germinação e instalação das plantas (Thompson 1974b), o que é atribuído como superação de dormência (Bewley \& Black 1982). O mecanismo metabólico de ativação da germinação deve estar ligado a alterações das propriedades das membranas (Thompson 1974b) regidas em parte, pelo menos, com a variação de temperatura e sua interação com o fitocromo (Taylorson \& Hendricks 1972; Bewley \& Black 1994).

Ademais, as propriedades das membranas são influenciadas pelo estado de hidratação do solo (substrato) e a disponibilidade de $\mathrm{O}_{2}$, que diminui com a ocupação por água dos espaços livres do substrato e por aumento da temperatura, com diminuição de sua solubilidade em água (Comê \& Tissaqui 1973). Tanto as sementes de T. patens como as de $B$. antiacantha não germinam abaixo de $20^{\circ} \mathrm{C}$ quando a temperatura é constante. Os impedimentos parecem ser diferentes para as duas espécies, pois em temperaturas alternadas $\left(10-20^{\circ} \mathrm{C}\right)$ para T. patens há percentual razoável de germinação e, para $B$. antiacantha, quando uma das temperaturas é abaixo de $20^{\circ} \mathrm{C}$, não há germinação (ex. $15-25^{\circ} \mathrm{C}$ - Tab.1).

A promoção da germinação pela alternância de temperatura está bastante relacionada às respostas germinativas de presença ou ausência de luz (Thompson 1974a). Por questões operacionais, os pares de temperaturas alternas foram feitos com diferenças de $10^{\circ} \mathrm{C}$, sendo que pode haver respostas mais pronunciadas, especialmente em plantas de ambientes campestres ou de hábitos ruderais, como T. patens, se a alternância for realizada na ausência de luz e com maiores amplitudes (Thompson \& Grime 1983). T. patens responde ao estímulo de luz, sendo os percentuais de germinação mais altos a 20,25 ou mesmo $30^{\circ} \mathrm{C}$. A comparação global entre luz e escuro, sem considerar o fator temperatura, foi significativamente diferente pelo teste de Duncan. Lima \& Felippe (1988) já haviam verificado o fotoblastismo positivo das sementes de T. patens, planta ruderal ou invasora de cultivos. Não houve seleção das sementes, de forma que apenas cerca de $40 \%$ estavam viáveis (Tab. 1). Destas sementes viáveis, cerca de $65 \%$ germinaram na luz, sendo este dado semelhante a outra espécie da família, Portulaca oleracea, na qual foi obtido percentual aproximado de germinabilidade (Singh 1973) com sementes recém-colhidas, como foi o caso do presente estudo.

Usando substrato areia, os percentuais de germinação são semelhantes (dados não apresentados), revelando que estas três espécies com potencial farmacológico podem ser propagadas por sementes, desde que no manejo das culturas sejam observadas as condições de temperatura e luz requeridas para cada uma das espécies estudadas.

\section{Agradecimentos}

Aos botânicos Rodrigo G. Magalhães, Silvia Marondin e Miriam Eira, pela identificação taxonômica das espécies utilizadas. À CAPES e ao CNPq, pelas bolsas concedidas à primeira e ao segundo Autores, respectivamente. 


\section{Referências bibliográficas}

Benech -Arnold, R. L. \& Sanchez, R. A. 1995. Modeling weed seed germination. Pp. 545-566. In: J. Kigel \& G. Galili (Ed.), Seed development and germination. Marcel Dekker Inc, New York.

Bewley, J. D. \& Black, M. 1982. Seeds: Physiology of development and germination. vol. 2. Plenum Press, New York.

Bewley, J. D. \& Black, M. 1994. Seeds: Physiology of develepment and germination. 2. ed. Plenum Press, New York.

Brasil 1993. Regras para Análises de Sementes. Ministério da Agricultura e Reforma Agrária, Brasília.

Côme, D. \& Tissaqui, T. 1973. Interrelated effects of imbibition, temperature and oxigen on seed germination. Pp. 157-168. In: W. Heydecker (Ed.), Seed Ecology. Butterworth Co., London.

Ferreira, A. G. 1977. Araucaria angustifolia (Bert.) O. Ktze : germinação da semente e desenvolvimento da plântula. Tese de Doutorado. Universidade de São Paulo, São Paulo.

Ghersa, C. M.; Benech-Arnold, R. L. \& Martinez-Ghersa, M. A. 1992. The role of fluctuating temperatures in germination and establishment of Sorghum halepense. Regulation of germination at increasing depths. Functional Ecology 6: 460- 468.

Grabe, D. F. 1970. Tetrazolium testing handbook. Association of Official Seed Analyst, The Tetrazolium Testing Commitee, Zurich.

Heydecker, W. 1977. Stress and seed germination: an agronomic view. Pp. 237-282. In: A . Khan. (Ed.), The physiology and biochemistry of seed dormancy and germination. Elsevier, Amesterdam.

Koller, D. 1972. Environmental control of seed germination. Pp. 1-101. In: T. T. Kozlowsky (Ed.), Seed biology. Academic Press, New York.

Labouriau, L. G. \& Costa, J. A. F. 1976. Objetivos e instalações básicas de um laboratório de fisiologia vegetal. Academia Brasileira de Ciências, Rio de Janeiro.

Labouriau, L. G. 1983. A germinação das sementes. Secretaria-Geral da Organização dos Estados Americanos, Washington.

Lima, R. F. \& Felippe, G. M. 1988. Efeitos de luz e temperatura na germinação de Talinum patens. Pp. $15-$ 21 In: Anais do V Congresso da Sociedade Botânica de São Paulo, Botucatu.

Lopes, A. M. V. 1997. Plantas usadas na medicina popular do Rio Grande do Sul. Universidade Federal de Santa Maria, Santa Maria.

Magalhães, R. G. 1997. Plantas medicinais na região do Alto Uruguai RS: conhecimentos de João Martins Fiúza, "Sarampião". Dissertação de Mestrado. Universidade Federal do Rio Grande do Sul, Porto Alegre.

Mayer, A. M. \& Poljakoff-Mayber, A. 1989. The germination of seeds. Pergamon Press, Oxford.

Meira, M. S. 1997. Distribuição espacial e estrutura de populaçōes de bromélias terrestres em um mosaico de floresta e campo. Dissertação de Mestrado. Universidade Federal do Rio Grande do Sul, Porto Alegre.

Reitz, R. 1983. Bromeliáceas e a malária-bromélia endêmica. Flora Ilustrada Catarinense (Brom), 1559.

Singh, K. P. 1973. Effect of temperature and light on seed germination of two ecotypes of Portulaca oleracea L. New Phytologist 72: 289-295.

Sokal, R. S. \& Rohlf, F. J. 1981. Biometry. W. H. Freeman, San Francisco.

Taylorson, R. B. \& Hendricks, S. B. 1972. Interactions of light and a temperature shift on seed germination. Plant Physiology 49: 127-130.

Thompson, P. A. 1974a. Germination of Celery (Apium graveolens L.) in response to fluctuating temperatures. Journal of Experimental Botany 25(84): 156-163.

Thompson, P. A. 1974b. Effects of fluctuating temperatures on germination. Journal of Experimental Botany 25(84): 164-175.

Thompson, K. \& Grime, J. P. 1983. A comparative study of germination responses to diurnally-fluctuating temperatures. Journal of Applied Ecology 20: 141-156.

Zonta, E. P. \& Machado, A. A. sd. SANEST- Sistema de Análise Estatística. Universidade Federal de Pelotas, Pelotas. 